\title{
Cardiac magnetic resonance imaging in clinical practice ${ }^{*}$
}

\author{
Ressonância magnética cardíaca na prática clínica
}

\section{Adriana Dias Barranhas ${ }^{1}$, Alair Augusto S. M. D. dos Santos ${ }^{2}$, Otavio R. Coelho-Filho ${ }^{3}$, Edson Marchiori ${ }^{4}$, Carlos Eduardo Rochitte ${ }^{5}$, Marcelo Souto Nacif ${ }^{6}$}

Barranhas AD, Santos AASMD, Coelho-Filho OR, Marchiori E, Rochitte CE, Nacif MS. Cardiac magnetic resonance imaging in clinical practice. Radiol Bras. 2014 Jan/Fev; $47(1): 1-8$.

Abstract Objective: To evaluate and describe indications, mainly diagnoses and cardiac magnetic resonance imaging findings observed in clinical practice.

Materials and Methods: Retrospective and descriptive study of cardiac magnetic resonance performed at a private hospital and clinic in the city of Niterói, RJ, Brazil, in the period from May 2007 to April 2011.

Results: The sample included a total of 1000 studies performed in patients with a mean age of $53.7 \pm 16.2$ years and predominance for male gender (57.2\%). The majority of indications were related to assessment of myocardial perfusion at rest and under pharmacological stress (507/1000; 51\%), with positive results in 36.2\% of them. Suspected myocarditis was the second most frequent indication (140/ $1000 ; 14 \%)$, with positive results in $63.4 \%$ of cases. These two indications were followed by study of arrhythmias (116/1000; $12 \%)$, myocardial viability (69/1000; 7\%) and evaluation of cardiomyopathies (47/1000; $5 \%)$. In a subanalysis, it was possible to identify that most patients were assessed on an outpatient basis (58.42\%).

Conclusion: Cardiac magnetic resonance has been routinely performed in clinical practice, either on an outpatient or emergency/ inpatient basis, and myocardial ischemia represented the main indication, followed by investigation of myocarditis, arrhythmogenic right ventricular dysplasia and myocardial viability.

Keywords: Cardiac magnetic resonance; Health profile; Evidence-based medicine; Diagnostic methods - policies.

Resu mo Objetivo: Avaliar e descrever as indicações, os principais diagnósticos e os achados de imagem de ressonâncias magnéticas cardíacas realizadas na prática clínica.

Materiais e Métodos: Estudo descritivo e retrospectivo dos exames de ressonância magnética cardíaca realizados em um hospital e uma clínica particulares do município de Niterói, RJ, no período de maio de 2007 a abril de 2011.

Resultados: Um total de 1000 exames foi incluído, com pacientes apresentando média de idade de 53,7 \pm 16,2 anos e predomínio no sexo masculino (57,2\%). A maioria das indicações foi para pesquisa de isquemia miocárdica com estresse farmacológico (507/ 1000; 51\%), que teve resultado positivo em 36,2\% das avaliações. A pesquisa de miocardite (140/1000; 14\%) foi a segunda indicação mais frequente, com resultados positivos em $63,4 \%$ dos casos. Estas duas principais indicações foram seguidas de avaliação de arritmias (116/1000; 12\%), viabilidade miocárdica (69/1000; 7\%) e cardiomiopatias diversas (47/1000, 5\%). Em uma subanálise, foi possível identificar que a grande maioria dos exames foi realizada pela via ambulatorial $(58,42 \%)$.

Conclusão: A ressonância magnética cardíaca está sendo realizada de rotina na prática clínica, tanto via ambulatorial ou pela via emergêncial/intra-hospitalar, e a pesquisa de isquemia miocárdica foi a principal indicação, seguida de miocardite, displasia arritmogênica do ventrículo direito e viabilidade miocárdica.

Unitermos: Ressonância magnética cardíaca; Perfil de saúde; Medicina baseada em evidências; Exames médicos - políticas.

* Study developed at Universidade Federal do Rio de Janeiro (UFRJ), Rio de Janeiro, RJ, Brazil. The present article is part of the doctoral thesis of Dr. Adriana Dias Barranhas, by the Program of Post-graduation in Radiology at UFRJ. Financia support: Conselho Nacional de Desenvolvimento Científico e Tecnológico (CNPq).

1. Master, MD, Radiologist, Hospital das Clínicas de Niterói (HCN), Niterói, RJ, Brazil.

2. PhD, Associate Professor, Universidade Federal Fluminense (UFF), Niterói, RJ, Brazil.

3. PhD, MD, Cardiologist, Universidade Estadual de Campinas (Unicamp), Campinas, SP, Brazil.

4. PhD, Full Professor, Universidade Federal Fluminense (UFF), Niterói, RJ, Adjunct Coordinator, Program of Post-Graduation in Radiology, Universidade Federal do Rio de Janeiro (UFRJ), Rio de Janeiro, RJ, Brazil.

5. PhD, Private Docent, Instituto do Coração do Hospital das Clínicas da Faculdade de Medicina da Universidade de São Paulo (InCor/HC-FMUSP), São Paulo, SP, Brazil.

6. PhD, Associate Professor, Universidade Federal Fluminense (UFF), Niterói, RJ, Brazil.

\section{INTRODUCTION}

Cardiac magnetic resonance imaging (cardiac MRI) is a noninvasive cardiovascular investigation method with the greatest technical-scientific development over the last thirty years ${ }^{(1)}$. Currently, cardiac MRI is routinely utilized at different health centers ${ }^{(2)}$ and the most frequent indications include the investigation of cardiomyopathies; myocarditis; stratification of risk for coronary artery disease (investigation of myocardial ischemia under pharmacological stress); and evaluation of myocardial viability, among others ${ }^{(3-10)}$.

Mailing Address: Dr. Marcelo Souto Nacif. Rua Barão de Cocais, 324, Bosque Imperial. São José dos Campos, SP, Brazil, 12242-042. E-mail: msnacif@gmail.com.

Received June 5, 2013. Accepted after revision October 20, 2013. 
The centers qualified to perform such procedure require intensive job training and an appropriate technology park. Different entities, such as American College of Cardiology Foundation Task Force ${ }^{(11)}$ and Society for Cardiovascular Magnetic Resonance ${ }^{(12,13)}$ have their experts consensus statements to guide and aid in the provision of the best clinical management of patients. In Brazil, the first guidelines on the utilization of the method was published in $2006^{(14)}$ and, over the last years, the number of qualified health centers has increased in the country. However, scarce or almost no scientific information on the clinical utilization of such method is available in Brazil.

The present study was developed to describe the profile of the population studied with cardiac MRI, according to indications and origin of the requested studies, and also to indentify the studies results in the groups with left ventricle ejection fraction (LVEJ) $\geq 50 \%$ and $<50 \%$, with emphasis on the diagnosis and main imaging findings for each group.

\section{MATERIALS AND METHODS}

\section{Patients selection}

The present descriptive and retrospective study was approved by the Committee for Ethics in Research of Hospital Universitário Clementino Fraga Filho - Universidade Federal do Rio de Janeiro (HUCFF-UFRJ) under No. 046/1 1CEP. In the period from May 2007 to April 201 1, one thousand cardiac MRI studies were performed at two private health centers in Niterói, RJ, Brazil, namely, Hospital de Clínicas de Niterói (HCN) and Pro-Echo - Niterói unit (PEN).

\section{Cardiac MRI protocol}

At both institutions, the routine follows a single protocol. Since the patients understanding and cooperation are extremely important, they are given an explanation on the procedure they will undergo as a routine standard pre-examination method to achieve the best quality possible. All the patients signed a term of free and informed consent, upon the explanation about the procedure and about the possible involved risks.

The images were acquired with the patients positioned in dorsal decubitus, remaining static and with their arms aligned along the body, and with breath-hold at the end of expiration as necessary.

At HCN, the studies were performed in a 1.5 tesla Magnetom Symphony ${ }^{\circledR}$ equipment (Siemens Medical Solutions; Germany), and at Pro-Echo Niterói, in a 1.5 tesla Signa GE Horizon ${ }^{\circledR}$ (General Electric; USA), both with a four-element cardiac phased-array coil and cardiac gating.

Paramagnetic contrast medium was utilized in all the studies - gadoversetamide (Optimark ${ }^{\circledR} ;$ Mallinckrodt, USA) at PEN, and gadodiamide (Omniscan ${ }^{\circledR}$; GE Healthcare, USA) at HCN, at a dose of $0.2 \mathrm{ml} / \mathrm{kg}$ injected by means of an infusion pump at a rate of $5 \mathrm{ml} / \mathrm{s}$.

In cases where the protocol with pharmacological stress was indicated, dipyridamole (Persantin ${ }^{\circledR}$; Boehringer Ingel- heim, Germany) at a dose of $0.56 \mathrm{mg} / \mathrm{kg} / 4 \mathrm{~min}$ was utilized. Aminophylline (AM Hyfilina ${ }^{\circledR}$; Hypofarma, Brazil) at a dose of $250 \mathrm{mg}$ during two minutes was the pharmaceutical utilized to reverse the action of dipyridamole.

\section{Clinical data analysis and cardiac MRI studies review}

The patients were divided into nine age groups $(<19$, 20-29, 30-39, 40-49, 50-59, 60-69, 70-79, 80-89, 90-99 years).

The clinical data and the images were analyzed for the whole study population and for the subgroups created according to the LVEF value.

The main indications were divided into groups according to the guidelines of Sociedade Brasileira de Cardiologia (SBC) (Brazilian Society of Cardiology) ${ }^{(14)}$.

All the images were processed as DICOM (digital imaging and communications in medicine) files and transferred to commercially available workstations (ADW 4.3; GE Medical Systems, USA, and Leonardo; Siemens Medical Solutions, Germany).

The diameter of great vessels, systolic and diastolic diameters of the left ventricle (LV), LVEF, length of the right ventricle (RV) and left atrium (LA), besides the presence or absence of late contrast enhancement after paramagnetic contrast injection, were evaluated at cardiac MRI. All the images were independently reviewed by two specialists and the discordant cases were solved by consensus.

\section{Statistical analysis}

The continuous variables are expressed as mean \pm standard deviation, and the categorical variables, as number and percentage, according to the situation. The statistical difference between groups with LVEF $\geq 50 \%$ and LVEJ $<50 \%$ was evaluated with the non-paired $t$-test. The softwares Stata ${ }^{\circledR}$, version 12.0 (StataCorp LP College Station; USA) and Excel plug-in (Daniel's XL Toolbox, version 4.01; USA) were utilized for statistical analysis, and $<0.05$ was considered as level of statistical significance.

\section{RESULTS}

The mean examination time was approximately $42.3 \pm$ 6.6 minutes.

The mean age of the study population was $53.7 \pm 16.2$ years, with no statistically significant difference between groups with LVEF $\geq 50 \%$ or $<50 \%(p=0.07)$. The male sex prevailed in the present study $(57.2 \%)$.

Table 1 describes the distribution of the patients submitted to cardiac MRI according to their age range. The patients' age ranged from two to 92 years. Amongst the proposed age ranges, a progressive increase was observed as from the fifth decade and highest prevalence at the sixth and seventh decades of life where the number of studies performed starts decreasing again. As the age range is analyzed in relation to sex, a male predominance was observed up to the seventh decade of life. 
Table 1-Distribution of patients submitted to cardiac MRI according to age range.

\begin{tabular}{lccc}
\hline & Male & Female & Total \\
\hline$\leq 19$ years & $21(3.6 \%)$ & $14(3.2 \%)$ & $35(3.5 \%)$ \\
20-29 years & $30(5.3 \%)$ & $22(5.2 \%)$ & $52(5.2 \%)$ \\
30-39 years & $65(11.4 \%)$ & $35(8.1 \%)$ & $100(10 \%)$ \\
40-49 years & $86(15.0 \%)$ & $73(17.1 \%)$ & $159(15.9 \%)$ \\
$50-59$ years & $156(27.3 \%)$ & $115(26.8 \%)$ & $271(27.1 \%)$ \\
$60-69$ years & $129(22.5 \%)$ & $86(20.0 \%)$ & $215(21.5 \%)$ \\
$70-79$ years & $62(10.9 \%)$ & $62(14.5 \%)$ & $124(12.4 \%)$ \\
$80-89$ years & $23(4.0 \%)$ & $19(4.5 \%)$ & $42(4.2 \%)$ \\
$90-99$ years & $0(0 \%)$ & $2(0.6 \%)$ & $2(0.2 \%)$ \\
Total & $572(100 \%)$ & $428(100 \%)$ & $1000(100 \%)$ \\
\hline
\end{tabular}

Out of the 1000 studies performed, $2(0.2 \%)$ were interrupted - in a 71-year-old woman and a 68-year-old man , both because of significant chest pain after administration of the stressor agent, but both patients evolved well after the procedure. In this period, neither reaction nor complications due to the use of contrast agent were observed.

Table 2 characterizes the study population and identifies clinical and imaging data for the whole population and for the subgroups according LVEF values. There is a statistically significant difference $(p<0.001)$ between the groups with $\mathrm{LVEF} \geq 50 \%$ or $<50 \%$ for diameters of the great vessels; for evaluation of the global LV function; and for length of the LA. The shortest length of RV was the only parameter which did not demonstrate any statistically significant difference between groups $(p=0.14)$.

From the total of 405 (40\%) cardiac MRI studies with positive late contrast enhancement for fibrosis, infarction of inflammatory process as the late enhancement technique was utilized, $224(48 \%)$ were identified in the group with LVEF $<50 \%$. On the other hand, most of the patients with no alterations at the $595(60 \%)$ studies with the late myocardial enhancement technique were in the group with LVEF $\geq 50 \%$ (352;66\%).

In the present study, the main indication for cardiac MRI was investigation of myocardial ischemia with pharmacological stress (Figure 1), in a total of 507 studies (51\%), followed by diagnostic evaluation of myocarditis (Figure 2), with 140 studies (14\%), and investigation of arrhythmias, with 116 studies (12\%). Most studies (798; 79.8\%) were performed in a hospital environment, a majority also observed for the most debilitated patients, i.e., those with LVEF < $50 \%(413 ; 88.5 \%)$ (Table 3). In the subanalysis of the patients submitted to cardiac MRI in the hospital, the authors observed that, in most cases, the procedures were performed on an outpatient basis $(58,42 \%)$, and $41.58 \%$ on an emergency/inpatient basis.

Table 4 demonstrates the results per indication and per institution where the cardiac MRI procedures were per-

Table 2-Distribution and characterization of the population submitted to cardiac MRI.

\begin{tabular}{|c|c|c|c|c|}
\hline Demographic data & Total $(n=1000)$ & LVEF $\geq 50 \%(n=533)$ & LVEF $<50 \% \quad(n=467)$ & $p$-value * \\
\hline Age & $53.7 \pm 16.2$ & $52.7 \pm 16.2$ & $54.8 \pm 16.2$ & 0.07 \\
\hline Male & 572 (57.2\%) & 280 (52.5\%) & 292 (62.4\%) & 0.007 \\
\hline Height (cm) & $166 \pm 15.4$ & $165.6 \pm 14.6$ & $166.3 \pm 16.3$ & 0.56 \\
\hline Weight (kg) & $76.0 \pm 16.5$ & $75.1 \pm 15.4$ & $77.0 \pm 17.7$ & 0.14 \\
\hline Heart beats (bpm) & $71.9 \pm 14.5$ & $70.9 \pm 13.8$ & $73.2 \pm 15.2$ & 0.03 \\
\hline Arterial systolic pressure (mmHg) & $129.8 \pm 22.6$ & $131.8 \pm 23.7$ & $127.9 \pm 21.3$ & 0.04 \\
\hline Arterial diastolic pressure (mmHg) & $77.9 \pm 13.3$ & $79.2 \pm 13.2$ & $76.4 \pm 13.3$ & 0.01 \\
\hline \multicolumn{5}{|l|}{ Great vessels } \\
\hline - Aortic root & $2.7 \pm 0.4$ & $2.6 \pm 0.4$ & $2.9 \pm 0.4$ & $<0.001$ \\
\hline - Ascending aorta & $3.0 \pm 0.5$ & $2.9 \pm 0.4$ & $3.1 \pm 0.5$ & $<0.001$ \\
\hline - Descending aorta & $2.2 \pm 0.3$ & $2.1 \pm 0.3$ & $2.3 \pm 0.3$ & $<0.001$ \\
\hline - Pulmonary artery trunk & $2.6 \pm 0.4$ & $2.5 \pm 0.3$ & $2.7 \pm 0.4$ & $<0.001$ \\
\hline - Inferior vena cava & $1.7 \pm 0.4$ & $1.6 \pm 0.3$ & $1.8 \pm 0.5$ & $<0.001$ \\
\hline \multicolumn{5}{|c|}{ Global left ventricle function at cardiac MRI } \\
\hline - LVEF (\%) & $48.9 \pm 17.1$ & $62.8 \pm 7.2$ & $33.1 \pm 10.2$ & $<0.001$ \\
\hline - Final diastolic diameter (cm) & $5.1 \pm 0.8$ & $4.7 \pm 0.5$ & $5.4 \pm 1.0$ & $<0.001$ \\
\hline - Final systolic diameter (cm) & $3.5 \pm 1.0$ & $3.1 \pm 0.5$ & $4.1 \pm 1.3$ & $<0.001$ \\
\hline \multicolumn{5}{|l|}{ Right ventricular length at cardiac MRI } \\
\hline - Greatest $(\mathrm{cm})$ & $7.7 \pm 0.9$ & $7.5 \pm 0.9$ & $8.0 \pm 1.0$ & $<0.001$ \\
\hline - Shortest (cm) & $3.8 \pm 0.6$ & $3.8 \pm 0.5$ & $3.9 \pm 0.6$ & 0.14 \\
\hline \multicolumn{5}{|l|}{ Left atrium at cardiac MRI } \\
\hline - Anteroposterior length & $3.5 \pm 0.6$ & $3.4 \pm 0.6$ & $3.7 \pm 0.7$ & $<0.001$ \\
\hline \multicolumn{5}{|l|}{ Late enhancement at cardiac MRI } \\
\hline - Positive & 405 (40.5\%) & $181(34 \%)$ & $224(48 \%)$ & Nonapplied \\
\hline - Negative & 595 (59.5\%) & 352 (66\%) & $243(52 \%)$ & Nonapplied \\
\hline Total & 1000 (100\%) & 533 (100\%) & $467(100)$ & \\
\hline
\end{tabular}



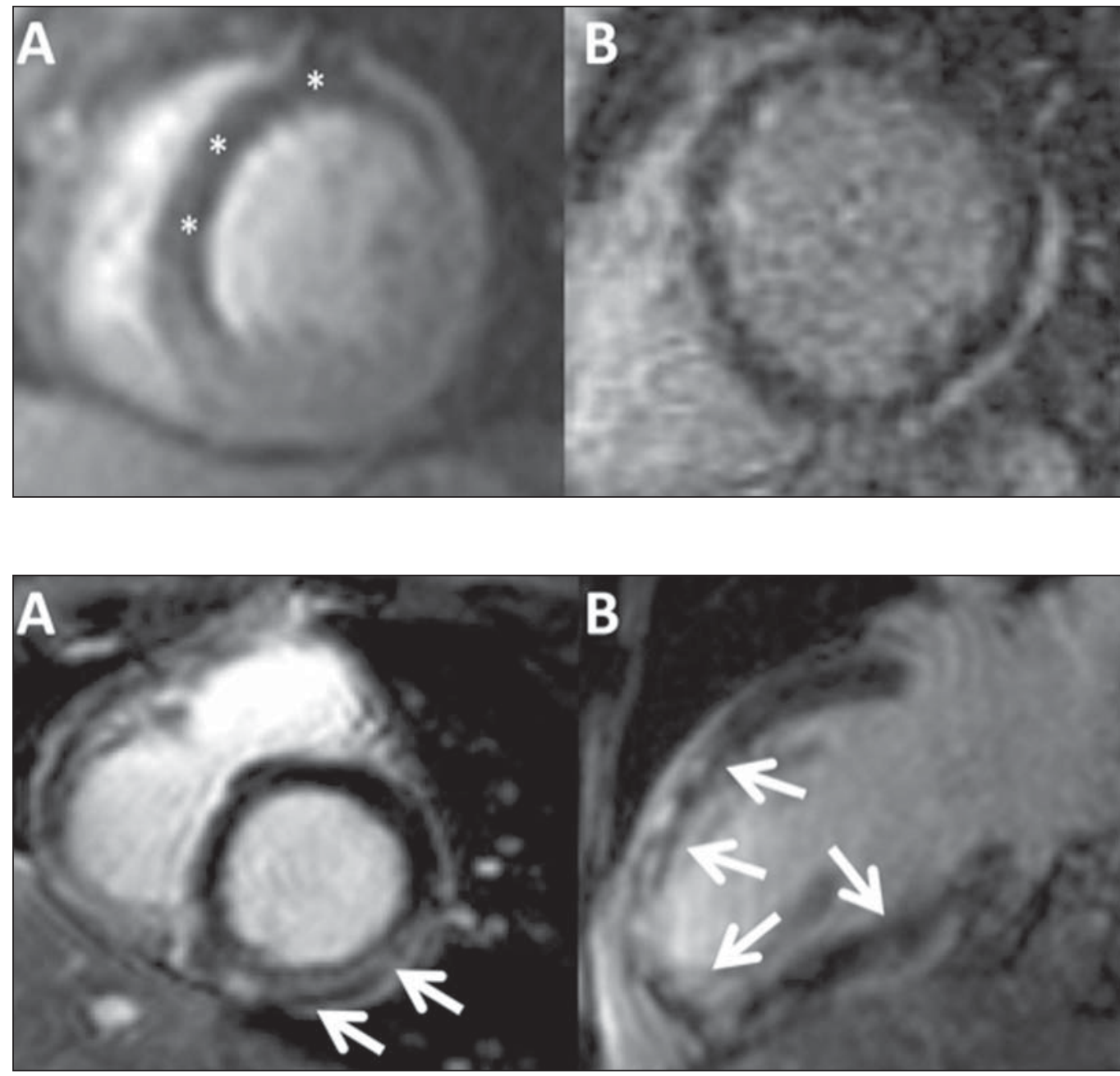

Figure 1. Male, 57-year-old patient. Perfusion study (A) during hyperemia induced by dipyridamole identified perfusional deffect (asterisk) in the region of the anterior descending coronary artery (black area). Late enhancement technique (B) demonstrated minimum subendocardial enhancement, corresponding to significant ischemia in the region of the anterior descending coronary artery. Catheterization demonstrated the presence of a severe lesion in the proximal anterior descending artery.

Table 3-Distribuion of cases according to indication and per institution.

\begin{tabular}{lccc}
\hline Indication & Total $(n=1000)$ & LVEF $\geq 50 \%(n=533)$ & LVEF < 50\% $(n=467)$ \\
\hline Investigation of ischemia & $507(51 \%)$ & $282(52.9 \%)$ & $225(48.2 \%)$ \\
Myocarditis & $140(14 \%)$ & $61(11.4 \%)$ & $79(16.9 \%)$ \\
Arrhythmias* & $116(12 \%)$ & $96(18.1 \%)$ & $20(4.4 \%)$ \\
Myocardial viability $_{\text {Investigation of ischemia and viability }}(79(7 \%)$ & $14(2.6 \%)$ & $47(10.1 \%)$ \\
Cardiomyopathies $^{\dagger}$ & $37(4 \%)$ & $15(2.9 \%)$ & $23(5 \%)$ \\
Hypertrophic cardiomyopathy & $47(5 \%)$ & $17(3.1 \%)$ & $32(6.8 \%)$ \\
Thrombus/tumor & $20(2 \%)$ & $7(1.3 \%)$ & $3(0.6 \%)$ \\
Congenital diseases & $13(1 \%)$ & $2(0.3 \%)$ & $6(1.2 \%)$ \\
Pericardial diseases & $11(1 \%)$ & $0(0 \%)$ & $9(1.8 \%)$ \\
Chest pain & $9(1 \%)$ & $5(1.0 \%)$ & $9(2 \%)$ \\
Others & $8(1 \%)$ & $12(2.3 \%)$ & $3(0.6 \%)$ \\
Institution & $23(2 \%)$ & $11(2.4 \%)$ \\
- Clinic & $202(20.2 \%)$ & $385(72.3 \%)$ & $54(11.5 \%)$ \\
- Hospital & $798(79.8 \%)$ & $413(88.5 \%)$ \\
\hline
\end{tabular}

Note: Results in figures and percentages.

* Arrhythmias: diagnostic ruling out of arrhythmogenic right ventricular dysplasia (89), investigation of arrhythmogenic substrate (fibrosis) (15), pre- and post-ablation study of atrial fibrillation (12).

${ }^{\dagger}$ Cardiomiopathies: dilated cardiomyopathies (37), restrictive cardiomyopathies (amyloidosis, 2; endomyocardial fibrosis, 2), peripartum cardiomyopathy (3), non compacted myocardium (2), Takotsubo (1).

¥ Others: collagenosis (5), morphostructural analysis RV/LV (4), sarcoidosis (3), subaortic subvalvular aneurysm (1), Gaucher’s disease (1), valvulopathy (3), Madelung syndrome (1), lipid myopathy (1), follow-up of pacopexy (2), surgical risk (2). 
Table 4-Distribution of indications and institutions where the cardiac MRI studies were performed, according to normal or altered findings (individual percentage analysis).

\begin{tabular}{|c|c|c|c|}
\hline Indication & Normal & Altered & Total \\
\hline Investigation of ischemia & 324 (63.8\%) & $183(36.2 \%)$ & 507 (100\%) \\
\hline Myocarditis & 51 (36.6\%) & 89 (63.4\%) & 140 (100\%) \\
\hline Arrhythmogenic right ventricular dysplasia & 85 (95.5\%) & 4 (4.5\%) & $89(100 \%)$ \\
\hline Other arrhythmias & $10(37 \%)$ & $17(63 \%)$ & $27(100 \%)$ \\
\hline Myocardial viability & $12(18 \%)$ & $57(82 \%)$ & 69 (100\%) \\
\hline Investigation of ischemia and viability & $6(14.8 \%)$ & 31 (85.2\%) & 37 (100\%) \\
\hline Cardiomyopathies* & 7 (14.7\%) & 40 (85.3\%) & 47 (100\%) \\
\hline Hypertrophic cardiomyopathy & $3(14.3 \%)$ & $17(85.7 \%)$ & $20(100 \%)$ \\
\hline Thrombus/tumor & $1(11.1 \%)$ & $12(88.9 \%)$ & $13(100 \%)$ \\
\hline Congenital diseases & $2(14,3 \%)$ & $9(85.7 \%)$ & $11(100 \%)$ \\
\hline Pericardial diseases & $0(0 \%)$ & 9 (100\%) & 9 (100\%) \\
\hline Chest pain & $1(16.7 \%)$ & 7 (83.3\%) & $8(100 \%)$ \\
\hline Others $^{\dagger}$ & $4(17.6 \%)$ & $19(82.4 \%)$ & $23(100 \%)$ \\
\hline \multicolumn{4}{|l|}{ Institution } \\
\hline - Clinic & $91(45.2 \%)$ & $111(54.8 \%)$ & $202(100 \%)$ \\
\hline - Hospital & 416 (52.1\%) & 382 (47.9\%) & 798 (100\%) \\
\hline
\end{tabular}

Note: Results in figures and percentages.

* Cardiomiopathies: dilated cardiomyopathies (37), restrictive cardiomyopathies (amyloidosis, 2; endomyocardial fibrosis, 2), peripartum cardiomyopathy (3), non compacted myocardium (2), Takotsubo (1).

${ }^{\dagger}$ Others: collagenosis (5), morphostructural analysis RV/LV (4), sarcoidosis (3), subaortic subvalvular aneurysm (1), Gaucher's disease (1), valvulopathy (3), Madelung syndrome (1), lipid myopathy (1), follow-up of pacopexy (2), surgical risk (2).

formed. As the findings are stratified according the indications, it was possible to identify that most patients $(63.8 \%)$ did not present any alteration in their myocardial perfusion in cases where the study was indicated for investigation of myocardial ischemia with pharmacological stress. In cases of indication for investigation of myocardial viability, 57 $(82 \%)$ studies indicated absence of viability in at least one segment. In the investigation of myocarditis, $89(63.4 \%)$ studies presented signs indicative of inflammatory process in the analysis of late enhancement - 76 (85.9\%) studies as initial diagnostic study, and $13(14.1 \%)$ as follow-up studies. One of such patients presented images compatible with Chagas disease, which was later confirmed by laboratory tests.

Out of the 116 studies performed to evaluate arrhythmia, $89(76.7 \%)$ were referred for investigation of arrhythmogenic LV dysplasia (ALVD), and among them 85 (95.5\%) did not present either major or minor signs of ARVD (Table 4), 3 had isolated RV dilatation (a minor criterion) with normal function, and 1 presented two major criteria according to the Task Force ${ }^{(15)}$.

In the present study, the authors have found 13 different types of cardiac masses (Figure 3). Thrombus and atrial myxoma were the most frequently found masses, with $38.4 \%$ and $15.4 \%$, respectively, amongst the cases referred for study (Table 5).

\section{DISCUSSION}

The present study reviewed one thousand cardiac MRI studies routinely performed according clinical indication in the city of Niterói, RJ, and demonstrated that the method is included as an aid in the daily clinical practice of assisting physicians, both on outpatient and on emergency/inpatient

Figure 3. Female, 62-year-old patient with an explansile mass in the right ventricular outflow tract. ECG gating acquisitions, short axis plane (A) and right ventricular outflow tract plane (B) demonstrating expansile mass with a pedicle attached to the free wall of the RV (arrows), adjacent to the ventricular band insertion, with characteristics of benignity, suggesting myxoma.

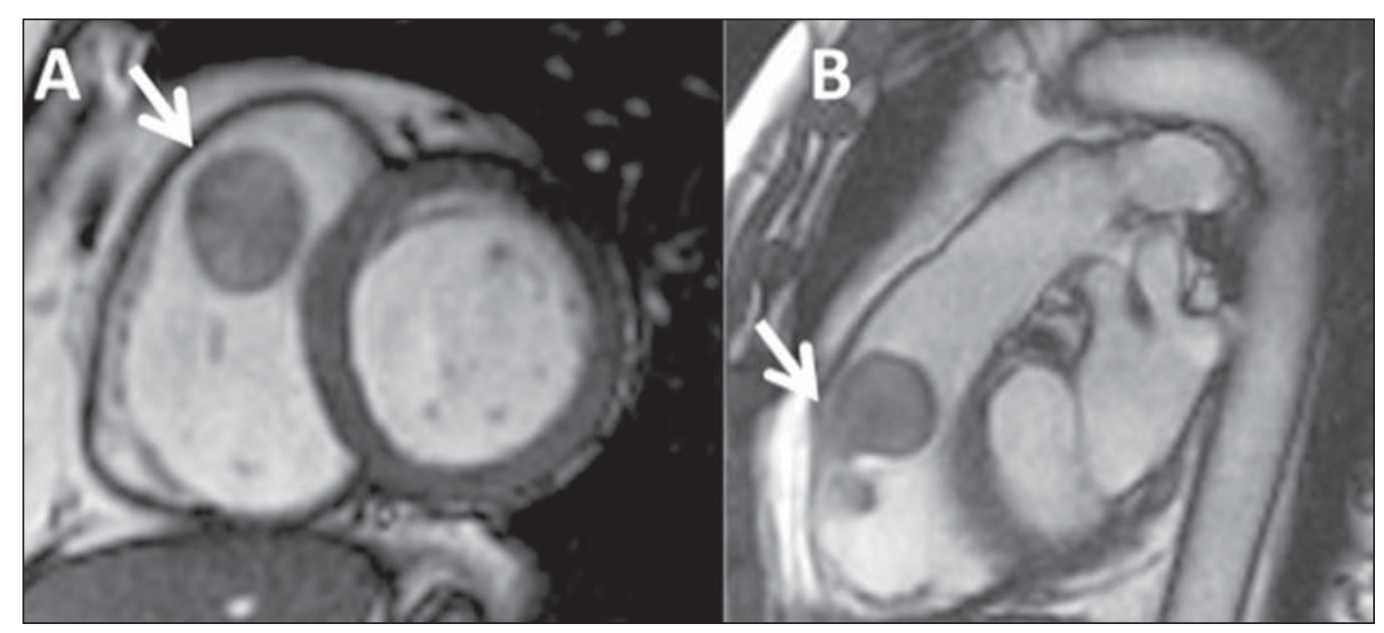


Table 5-Diagnostic findings in patients referred for evaluation of cardiac mass identified at echocardiography.

\begin{tabular}{lc}
\hline Findings & Frequency \\
\hline Thrombus & $5(38.4 \%)$ \\
Atrial myxoma & $2(15.4 \%)$ \\
Cardiac rhabdomyoma & $1(7.7 \%)$ \\
Lung tumor invading the right atrium & $1(7.7 \%)$ \\
Lesion suggestive of metastatic implant in right ventricle & $1(7.7 \%)$ \\
Pericardial cyst & $1(7.7 \%)$ \\
Lipid hyperplasia of interatrial septum & $1(7.7 \%)$ \\
Normal & $1(7.7 \%)$ \\
Total & $13(100 \%)$ \\
\hline
\end{tabular}

bases. Additionally, the authors observed that, in the region, cardiac MRI has been utilized in patients of all age groups, and that it is most useful in the daily clinical practice to investigate, among others, myocardial ischemia under pharmacological stress and patients under suspicion of myocarditis

Currently, cardiac MRI is considered the gold standard in the quantification of cardiac mass and volume, because of its low variability, high reproducibility and better accuracy as compared with other imaging methods utilized in cardiology ${ }^{(8,16)}$. However, the stratification of the study population according to LVEJ values is rather for illustrative purposes because of the population heterogeneity. It should be highlighted that the main imaging marker with solid scientific basis for follow-up and prognostic evaluation of the general population is the quantification of the LV mass, as evidenced by the Multi-Ethnics Study of Atherosclerosis $(\mathrm{MESA})^{(17,18)}$.

In the present study, considering the male predominance $(57.2 \%)$, the age range of $53.7 \pm 16.2$ years, and the high number of indications for stratification of cardiovascular risk, the authors have observed that many MESA propositions ${ }^{(19)}$ may be applied to the present population benefiting from cardiac MRI in the region. In Brazil, the Brazilian Longitudinal Study of Adult Health (ELSA-Brazil) ${ }^{(20)}$ must bring information appropriate to the needs of our population and hopefully with specific data little different from data previously published in international studies.

Cardiac MRI is performed with high safety and excellent effectiveness ${ }^{(21,22)}$ in the assessment of patients with ischemia and cardiopathies. In the present study, the authors have not observed any complication resulting from the utilization of paramagnetic contrast medium (gadolinium) and no patient died because of the procedure. Only two procedures were interrupted upon the patients' request because of chest pain after administration of dipyridamole. Most complications of cardiac MRI are related to the utilization of pharmacological stress and the safety of such a procedure is similar to the one of echocardiography and scintigraphy with pharmacological stress and cardiac stress test ${ }^{(23)}$.

As the main indications are identified and compared with the Brazilian guidelines, all of them are classified as I and IIa - investigation of myocardial ischemia (IIa), investiga- tion of myocardial viability (I), investigation of myocarditis (IIa) and ARVD $(\mathrm{I})^{(14)}$. It is the authors' opinion that some of such classifications must be revised and updated in the next version of the guidelines with a view on the clinical impact on the therapeutic decision. As the authors' institution is not a reference in the study of congenital cardiopathies, there was a poor utilization of the method, but its relevance in this selected group of patients should be highlighted. Even so, the authors can in general highlight that the indications are in compliance with the guidelines and that the referred patients are potentially benefiting from the utilization of this noninvasive imaging diagnosis method.

The investigation of ischemia by the myocardial perfusion technique, both at rest and after dipyridamole infusion, has provided good diagnostic accuracy as compared with invasive cinecoronariography, positron emission tomography (PET) and myocardial perfusion scintigraphy (SPECT) $)^{(22-}$ ${ }^{24)}$. For this reason, in some centers, it has been an alternative method to myocardial scintigraphy in the evaluation of coronary disease (class IIa, according to the guidelines), but, in the authors' institution, it is the method of choice, whose diagnostic superiority has already been scientifically proved $^{(11,22,25)}$. It is useless to be focused only on literature data and, for this reason, in a recent publication ${ }^{(21)}$, the authors demonstrated that their results are comparable to the ones in the international literature.

It should be highlighted that the high number of cardiac MRI studies performed for the investigation of myocardial ischemia with pharmacological stress is not necessarily the experience of most centers which utilize this method. The authors are describing the experience of a center where nuclear medicine nuclear is not available and, for this reason, since 2007, the use of cardiac MRI has prevailed, as applicable. However, it is important to highlight that there are innumerable studies demonstrating the diagnostic superiority of cardiac MRI over SPECT ${ }^{(20,24)}$, thus it is the authors' opinion that the experience reported herein may be utilized in other services of this speciality to improve the management of cardiovascular diseases.

The evaluation of late myocardial enhancement is still useful in the investigation of myocarditis, arrhythmias, myocardial viability and cardiomyopathies. With such a technique, cardiac MRI has become a unique method as compared with other imaging methods in the quantification of myocardial fibrosis. Some studies have already demonstrated that the amount of fibrosis and the degree of myocyte degeneration are inversely proportional to the systolic ${ }^{(26)}$ and diastolic $^{(27)}$ functions of the LV. Also, it has already been demonstrated that the quantification of fibrosis by the late enhancement technique at cardiac MRI has prognostic significance in patients with severe aortic valve disease ${ }^{(28)}$. Such facts reinforce the diagnostic and prognostic role of cardiac MRI in the clinical practice.

The late enhancement technique has shown to be superior to myocardial perfusion scintigraphy in the evaluation 
of myocardial viability, with the same sensitivity and specificity of PET so cardiac MRI is the method of choice for such purpose (class I according to the guidelines) $)^{(13,14)}$. In the present study, amongst the 69 patients referred specifically for evaluation of myocardial viability, lack of potential for myocardial contractile recovery (viability) ${ }^{(29,30)}$ was observed in at least one segment in $82 \%$ of cases. It is important to reinforce that such figures will be higher if patients submitted to pharmacological stress and investigation for myocardial viability are included in a single protocol. This just demonstrates that the indication for the method is appropriate.

Myocarditis was isolatedly the second major indication for cardiac MRI (class IIa according to the guidelines) ${ }^{(11)}$. Many studies reveal the relevance of cardiac MRI in the diagnosis, both in the acute and in the chronic phases of myocarditis $^{(31,32)}$. The multifocal myocardial enhancement pattern has specificity of $96 \%$ and sensitivity of $76 \%$ in the diagnosis, so cardiac MRI is one of the first choices in the investigation of myocarditis ${ }^{(32)}$. In the present study, little more than half of patients referred with such an indication presented imaging findings compatible with myocarditis. Cardiac MRI was also indicated to follow-up nine patients with myocarditis, all of them with improvement of imaging findings, and one with complete remission, i.e. late enhancement became negative. The prevalence of myocarditis could not yet be established due to the difficulty in confirming a diagnosis by means of invasive methods, which will certainly change with the utilization of cardiac MRI ${ }^{(33)}$.

The diagnostic ruling out of ARVD or investigation of arrhythmogenic substrate and pre- and post-ablation study, particularly in cases of atrial fibrillation, also represent good indications in the clinical practice ${ }^{(34)}$. The present study data reflect the broad utilization of the method as indicated.

Patients with hypertrophic cardiomyopathy ${ }^{(35)}$ also benefit from cardiac MRI, not only because of its potential for anatomical mapping and fibrosis quantification, but also for prognostic stratification of such patients ${ }^{(36)}$. In the present study, the authors observed that $2 \%$ of all the cardiac MRI studies were related to the investigation of hypertrophic cardiomyopathy.

Primary tumors of the heart are not frequently found, with an incidence at necropsy ranging between $0.0017 \%$ and $0.28 \%^{(37)}$. Only two confirmed cases with characteristics suggestive of myxomas were found in the present study. One of them was located in the right ventricular outflow tract. The main differential diagnosis among cardiac tumors is made with thrombi, and most of times, cardiac MRI can make such a differentiation.

With the present study, the authors have demonstrated that, in the daily clinical practice, cardiac MRI offers detailed information on several clinical conditions, particularly in the stratification of cardiovascular risk with investigation of myocardial viability and ischemia with perfusion under stress after use of coronary vasodilator which are already validated as cardiac events predictors ${ }^{(38)}$.

\section{CONCLUSION}

The population studied with cardiac MRI is quite heterogeneous, covering all the age ranges and a wide spectrum of clinical indications. The most prevalent indications include investigation of myocardial ischemia, followed by investigation of myocarditis, arrhythmogenic left ventricular dysplasia and myocardial viability. However, and not less important, many types of dilated and hypertrophic cardiomyopathies, arrhythmogenic and congenital diseases, besides cardiac tumors, can be investigated.

Cardiac MRI has been routinely performed in the clinical practice, according to appropriate criteria established by the current guidelines, both on outpatient and on emergency/ inpatient bases.

\section{Acknowledgement}

To the clinical team of Medical Practice, Clinical and Intensivist Cardiology, besides the team of Cardiac Surgery and Imaging Diagnosis of Hospital de Clínicas de Niterói, currently HCNcardiovascular.

\section{REFERENCES}

1. Higgins CB, Botvinick EH, Lanzer P, et al. Cardiovascular imaging with nuclear magnetic resonance. Cardiol Clin. 1983;1:527-39.

2. Bruder O, Schneider S, Nothnagel D, et al. EuroCMR (European Cardiovascular Magnetic Resonance) registry: results of the German pilot phase. J Am Coll Cardiol. 2009;54:1457-66.

3. Nacif MS, Falcão RO, Carvalhal AJS. Anomalia de Ebstein com fibrose septal. Radiol Bras. 2011;44(5):ix-xi.

4. Nacif MS, Kawel N, Sibley CT, et al. Infarto do miocárdio por uso de cocaína, associado com piora do strain global e elevação do volume extracelular calculado pelo mapa T1. Radiol Bras. 2011;44(2):xi-xiii.

5. Nacif MS, Noureldin RAA, Sibley CT, et al. Insuficiência cardíaca com análise de strain e caracterização de tecido fibrótico (mapa T1). Radiol Bras. 2010;43(5):xi-xiii.

6. Nacif MS, Santos TCCRS, Huang J, et al. Ventrículo esquerdo com dupla câmara. Radiol Bras. 2010;43(4):ix-xi.

7. Nacif MS, Oliveira Junior AC, Falcão RO, et al. Cardiopatia induzida por estresse (balonamento apical transitório do ventrículo esquerdo, síndrome do coração partido ou síndrome de Takotsubo) Radiol Bras. 2010;43(2):ix-xi.

8. Nacif MS, Marchiori E, Rochitte CE. Ressonância magnética cardíaca para radioablação de fibrilação atrial: protocolo e técnicas de quantificação do volume atrial esquerdo. Radiol Bras. 2009;42:370.

9. Nacif MS, Côrtes DCS, Oliveira Junior AC, et al. Hipoplasia do ventrículo direito associada a comunicação interatrial e estenose supravalvar do tronco da pulmonar em paciente adulto. Radiol Bras. 2009;42(5):xi-xiv.

10. Nacif MS, Cavalieri BC, Oliveira Junior AC, et al. Mixoma de átrio direito. Radiol Bras. 2009;42(1):ix-xi.

11. Hundley WG, Bluemke DA, Finn JP, et al. ACCF/ACR/AHA/ NASCI/SCMR 2010 expert consensus document on cardiovascular magnetic resonance: a report of the American College of Cardiology Foundation Task Force on Expert Consensus Documents. J Am Coll Cardiol. 2010;55:2614-62.

12. Kramer CM, Barkhausen J, Flamm SD, et al. Standardized cardiovascular magnetic resonance imaging (CMR) protocols, society for cardiovascular magnetic resonance: board of trustees task force on standardized protocols. J Cardiovasc Magn Reson. 2008;10:35.

13. Hundley WG, Bluemke D, Bogaert JG, et al. Society for Cardiovascular Magnetic Resonance guidelines for reporting cardiovascu- 
lar magnetic resonance examinations. J Cardiovasc Magn Reson. 2009;11:5.

14. Rochitte CE, Pinto IM, Fernandes JL, et al. I cardiovascular magnetic resonance and computed tomography guidelines of the Brazilian Society of Cardiologia - Executive summary. Arq Bras Cardiol. 2006;87:e48-59.

15. Marcus FI, McKenna WJ, Sherrill D, et al. Diagnosis of arrhythmogenic right ventricular cardiomyopathy/dysplasia: proposed modification of the task force criteria. Circulation. 2010;121:1533-41.

16. Omoumi P, Métais JP, Bertrand P, et al. Left and right ventricular volumetry and ejection fraction with MRI: segmentation criteria and interobserver reproducibility. J Radiol. 2010;91:769-78.

17. Polak JF, Wong Q, Johnson WC, et al. Associations of cardiovascular risk factors, carotid intima-media thickness and left ventricular mass with inter-adventitial diameters of the common carotid artery: the Multi-Ethnic Study of Atherosclerosis (MESA). Atherosclerosis. 2011;218:344-9.

18. Bluemke DA, Kronmal RA, Lima JA, et al. The relationship of left ventricular mass and geometry to incident cardiovascular events: the MESA (Multi-Ethnic Study of Atherosclerosis) study. J Am Coll Cardiol. 2008;52:2148-55.

19. Liu S, Bluemke DA. Multicenter epidemiological studies of atherosclerosis imaging. Top Magn Reson Imaging. 2009;20:239-46.

20. Aquino EM, Barreto SM, Bensenor IM, et al. Brazilian Longitudinal Study of Adult Health (ELSA-Brasil): objectives and design. Am J Epidemiol. 2012;175:315-24.

21. de Mello RA, Nacif MS, dos Santos AA, et al. Diagnostic performance of combined cardiac MRI for detection of coronary artery disease. Eur J Radiol. 2012;81:1782-9.

22. Nandalur KR, Dwamena BA, Choudhri AF, et al. Diagnostic performance of stress cardiac magnetic resonance imaging in the detection of coronary artery disease: a meta-analysis. J Am Coll Cardiol. 2007;50:1343-53.

23. Lee TH, Boucher CA. Clinical practice. Noninvasive tests in patients with stable coronary artery disease. N Engl J Med. 2001;344: 1840-5.

24. Schwitter J, Wacker CM, Wilke N, et al. Superior diagnostic performance of perfusion-cardiovascular magnetic resonance versus SPECT to detect coronary artery disease: The secondary endpoints of the multicenter multivendor MR-IMPACT II (Magnetic Resonance Imaging for Myocardial Perfusion Assessment in Coronary Artery Disease Trial). J Cardiovasc Magn Reson. 2012;14:61.

25. Schwitter J, Wacker CM, Wilke N, et al. MR-IMPACT II: Magnetic Resonance Imaging for Myocardial Perfusion Assessment in Coronary artery disease Trial: perfusion-cardiac magnetic resonance vs. single-photon emission computed tomography for the detec- tion of coronary artery disease: a comparative multicentre, multivendor trial. Eur Heart J. 2013;34:775-81.

26. Hein S, Arnon E, Kostin S, et al. Progression from compensated hypertrophy to failure in the pressure-overloaded human heart: structural deterioration and compensatory mechanisms. Circulation. 2003;107:984-91.

27. Villari B, Vassalli G, Monrad ES, et al. Normalization of diastolic dysfunction in aortic stenosis late after valve replacement. Circulation. 1995;91:2353-8.

28. Azevedo CF, Nigri M, Higuchi ML, et al. Prognostic significance of myocardial fibrosis quantification by histopathology and magnetic resonance imaging in patients with severe aortic valve disease. J Am Coll Cardiol. 2010;56:278-87.

29. Azevedo Filho CF, Hadlich M, Petriz JL, et al. Quantification of left ventricular infarcted mass on cardiac magnetic resonance imaging: comparison between planimetry and the semiquantitative visual scoring method. Arq Bras Cardiol. 2004;83:118-24;111-7.

30. Kim RJ, Wu E, Rafael A, et al. The use of contrast-enhanced magnetic resonance imaging to identify reversible myocardial dysfunction. N Engl J Med. 2000;343:1445-53.

31. Deux JF, Maatouk M, Lim P, et al. Acute myocarditis: diagnostic value of contrast-enhanced cine steady-state free precession MRI sequences. AJR Am J Roentgenol. 2011;197:1081-7.

32. Kindermann I, Barth C, Mahfoud F, et al. Update on myocarditis. J Am Coll Cardiol. 2012;59:779-92.

33. Olimulder MA, van Es J, Galjee MA. The importance of cardiac MRI as a diagnostic tool in viral myocarditis-induced cardiomyopathy. Neth Heart J. 2009;17:481-6.

34. Godeschalk-Slagboom CJ, van der Geest RJ, Zeppenfeld K, et al. Cardiac MRI visualization for ventricular tachycardia ablation. Int J Comput Assist Radiol Surg. 2012;7:753-67.

35. Vogel-Claussen J, Santaularia Tomas M, Newatia A, et al. Cardiac MRI evaluation of hypertrophic cardiomyopathy: left ventricular outflow tract/aortic valve diameter ratio predicts severity of LVOT obstruction. J Magn Reson Imaging. 2012;36:598-603.

36. Amano Y, Takayama M, Fukushima Y, et al. Delayed-enhancement MRI of apical hypertrophic cardiomyopathy: assessment of the intramural distribution and comparison with clinical symptoms, ventricular arrhythmias, and cine MRI. Acta Radiol. 2011;52:613-8.

37. Grizzard JD, Ang GB. Magnetic resonance imaging of pericardial disease and cardiac masses. Magn Reson Imaging Clin N Am. 2007; 15:579-607, vi.

38. Hundley WG, Morgan TM, Neagle CM, et val. Magnetic resonance imaging determination of cardiac prognosis. Circulation. 2002;106: $2328-33$. 